CLINICAL AND EXPERIMENTAL VACCINE RESEARCH

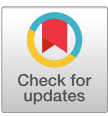

Clin Exp Vaccine Res 2019;8:110-115 https://doi.org/10.7774/cevr.2019.8.2.110 pISSN 2287-3651 • eISSN 2287-366X

\section{Rolando Felipe Ochoa Azze}

Department of Immunology, Institute of Basic and Preclinical Sciences, University of Medical Sciences, Havana, Cuba

Received: April 12, 2019

Revised: July 18, 2019

Accepted: July 22, 2019

Corresponding author: Rolando Felipe Ochoa Azze, $\mathrm{MD}, \mathrm{PhD}$

Department of Immunology, Institute of Basic and Preclinical Sciences, University of Medical Sciences, Ave. 146 No. 3102, Playa, Havana, Cuba Tel: +53-7271-9123, Fax: +53-5842-6717

E-mail: rochoa@infomed.sld.cu

No potential conflict of interest relevant to this article was reported.

The author thanks Mayelín Mirabal, MSc, for support with statistical analysis, and Oliver Pérez, $\mathrm{PhD}$, for his basic articles and other data used in this manuscript. The help of Eloísa Le Riverend, MSc, with the English version is also acknowledged.

\title{
A meningococcal $B$ vaccine induces cross-protection against gonorrhea
}

Purpose: Neisseria meningitidis and Neisseria gonorrhoeae share between $80 \%$ and $90 \%$ of their genetic sequence. Meningococcal serogroup $B$ vaccines based on outer membrane vesicles - such as VA-MENGOC-BC_could cross-protect against gonorrhea. The aim of this study was to analyze the incidence rates of gonorrhea and other sexually transmitted diseases with respect to the use of the VA-MENGOC-BC vaccine.

Materials and Methods: Health statistics between 1970 and 2017 were reviewed and the incidence of meningococcal disease and sexually transmitted diseases (gonorrhea, syphilis, condyloma acuminatum, hepatitis B and human immunodeficiency virus infection) were analyzed during the pre- and post-vaccination periods. Gonorrhea incidence was also analyzed by age groups.

Results: VA-MENGOC-BC was successfully used to control a meningococcal epidemic in Cuba. The strategy to combat the epidemic was carried out in two stages. The first one was a nationwide mass-vaccination campaign from 1989 to 1990, targeting the population at highestrisk aged 3 months to 24 years. During the second stage, begun in 1991, it was included in the Expanded Immunization Program. Gonorrhea incidence increased from 1970 to 1989. However, after the VA-MENGOC-BC massive vaccination campaign a sharp decrease of gonorrhea incidence was observed. It lasted between 1989 and 1993. A second incidence peak was detected in 1995, but it dropped again. Data clearly show a decline in the incidence of gonorrhea following massive vaccination, in contrast with other sexually transmitted diseases. Incidence rates in unvaccinated age groups also decreased, probably due to herd immunity.

Conclusion: There is evidence that VA-MENGOC-BC could induce a moderate protection against gonorrhea.

Keywords: Neisseria meningitidis, Neisseria gonorrhoeae, Gonorrhea, Vaccines

\section{Introduction}

Gonorrhea is a sexually transmitted disease, caused by Neisseria gonorrhoeae. Since the introduction of antibiotic treatment in the mid-30s of the last century, resistance to these antibacterial agents has increased [1]. On the other hand, the development of specific vaccines has been discouraging; no vaccine candidate has been able to induce protection against this disease [1-3]. Cross-protection against gonorrhea induced by meningococcal serogroup B outer membrane vesicle (OMV) vaccines has been reported in the last 2 years [1-5]. 
MeNZB is a vaccine based on OMVs. It was successfully used in New Zealand to control the epidemic outbreak caused by strain B:4:P1.7b,4. MeNZB effectiveness against strain specific disease in people from 6 months to 19 years old was finally estimated to be $68 \%$, and it was $56 \%$ against non-strain specific serogroup B meningococci, demonstrating protection beyond the PorA subtype [3]. Its effectiveness against gonorrhea was estimated as $31 \%$, according to a retrospective case-control study performed on individuals between 15 and 30 years of age [4].

A large epidemic of meningococcal disease affected Cuba during the 1980s and 1990s of the last century. It was caused by the hypervirulent strain B4:P1.19,15:L3,7,9. VA-MENGOC$\mathrm{BC}$ vaccine was developed to control this outbreak. It is a bivalent vaccine based on OMV from the epidemic strain and capsular polysaccharide of serogroup C meningococci [6-9].

The phase III efficacy trial of VA-MENGOC-BC was conducted in students aged 10 to 16 years during 1987. The estimated efficacy was $83 \%$. A similar estimate of $81 \%$ was achieved in a cohort study performed in Cuban children under 4 years old. Vaccine effectiveness against heterologous strains has been studied in other countries. It has been higher than $80 \%$ in individuals older than 4 years old, and about $60 \%$ in children under 4 years $[6,8,9]$.

Between 1989 and 1990 a massive vaccination campaign was carried out with VA-MENGOC-BC in the population group most affected by the epidemic outbreak of meningococcal disease in Cuba: from 3 months to 20 years of age, which was later extended to 24 years old. Vaccination coverage was $95 \%$, although some authors reported $75 \%$ [6-9].

In 1991, VA-MENGOC-BC was included in the Cuban Immunization Program, at 3 and 5 months of age. In addition, vaccination has been gradually extended to non-immunized people living in closed communities since 1995. Boosters are applied to young people during compulsory military service and in other high-risk groups [8,9].

After the massive immunization campaign carried out in Cuba between 1989 and 1990, a decrease in the incidence of gonorrhea was observed, especially by Perez et al. [7], the first researcher to describe this phenomenon. However, other sexually transmitted diseases have not been thoroughly studied. The analysis of their incidence rates is necessary to ascertain the actual impact of meningococcal vaccination on gonorrhea incidence rates.

The aim of this study was to analyze the incidence rates of gonorrhea and other sexually transmitted diseases, as well as meningococcal disease, with regards to the use of VA-MENGOC-BC vaccine during the epidemic and postepidemic periods.

\section{Materials and Methods}

\section{Morbidity of sexually transmitted diseases and meningococcal disease}

The morbidity of meningococcal disease, gonorrhea, syphilis, hepatitis B, and condyloma acuminatum was analyzed using the incidence rates per 100,000 inhabitants, published for each disease in the Time Series 1970 to 2013 by the National Directorate of Medical Records and Health Statistics of the Ministry of Public Health [10]. The information until 2017 was complemented with data reported in the Cuban Health Statistical Yearbook [11]. National reports and the Joint United Nations Programme on HIV and AIDS (UNAIDS) were used to analyze human immunodeficiency virus (HIV) infection rates [11-13].

\section{Evaluation of the incidence rates of sexually transmitted diseases regarding meningococcal vaccination}

Gonorrhea incidence rates were especially evaluated with respect to VA-MENGOC-BC vaccination: prevaccination (before 1989) and postvaccination periods. In the same way, other sexually transmitted diseases were analyzed as controls, taking into account that they share the same transmission route, but their biological agents are not genetically related to Neisseria. Gonorrhea incidence rates were also analyzed by age groups: <1, 1-4, 5-9, 10-14, 15-24, 25-64, and $\geq 65$ years old.

\section{Statistical analysis}

The correlate of protection ( $\mathrm{r}$ ) between meningococcal disease and gonorrhea was estimated after vaccination, as well as between gonorrhea and other sexually transmitted diseases. The Student's t test was used to evaluate the relationship between the variables, $\mathrm{p}<0.05$ was considered significant. Analyses were performed using Statgraphics Plus for Windows ver. 3.1 (Statistical Graphics Corp., Rockville, MD, USA).

A table was prepared to evaluate the incidence of meningococcal disease and sexually transmitted diseases in 1989, when mass-vaccination began, 5 years after the start of vaccination (1993), and the last year (2017) analyzed.

Combined line graphs were prepared. The graph that show the incidence rates of the total population for meningococcal disease, gonorrhea, syphilis, hepatitis B, HIV infection and 
condyloma acuminatum, was made with data available from 1970 to 2017. The graph corresponding to gonorrhea rates by age group was also made with available data, and covered the period from 1970 to 2013.

\section{Ethics}

VA-MENGOC-BC vaccine is manufactured by Finlay Institute of Vaccines, Havana, Cuba. Its scientific and ethics committees approved the study (code: 002-10-03-2018). Public data were used. No patient files or electronic medical records were accessed at any stage.

\section{Results}

Meningococcal disease morbidity maintained a downward trend, from $14.4 \times 100,000$ inhabitants in the epidemic period to $<0.1 \times 100,000$ inhabitants. This last incidence rate continues since 2008. Gonorrhea incidence decreased abruptly and parallel to meningococcal disease incidence from 1989 to 1993 (381.9 and 190.3 cases of gonorrhea $\times 100,000$ inhabitants, respectively) $(\mathrm{r}=0.9607, \mathrm{p}=0.001)$. Until 1995, it showed an upward trend again $(411.7 \times 100,000)$, and then decreased once more ( $\mathrm{r}=0.9501, \mathrm{p}=0.008)$ (Fig. 1) [10-12].
Negative correlation between gonorrhea and syphilis was found from 1989 to $1993(\mathrm{r}=-0.9755, \mathrm{p}=0.004)$. Syphilis incidence continued to rise until $1997(143.3 \times 100,000)$, contrasting with the sudden decrease of gonorrhea (Fig. 1) [10-12].

Condyloma acuminatum had lower rates and moderate upward trend until 2013 the last year in which data on this disease were published (Fig. 1) [10-12]. Negative correlation compared to gonorrhea was detected since 1995 (19.2 cases of condyloma acuminatum $\times 100,000)$ to $2013(36.3 \times 100,000)$ $(\mathrm{r}=-0.6506, \mathrm{p}=0.002)$.

Reports on hepatitis B and HIV infection began to be reported at the end of the 1980s. Hepatitis B rates increased until 1992 (20.3×100,000); then dropped (Fig. 1) [10-12]. However, negative correlation with respect to gonorrhea was found from 1989 to 1993 ( 14.9 and 17.5 cases of hepatitis $B \times 100,000$, respectively) ( $\mathrm{r}=-0.6172, \mathrm{p}=0.001)$. HIV incidence is low, although it has increased during the last years (Fig. 1) [10-13]. Negative correlation between gonorrhea and HIV was detected from 1989 to 1993 (1.2 and 1.8 cases of HIV $\times 100,000$, respectively) ( $\mathrm{r}=-0.6745, \mathrm{p}=0.001)$ and from 1995 to 2017 (2.1 and $17.0 \times 100,000)(\mathrm{r}=-0.7516, \mathrm{p}=0.0002)$.

The incidence of gonorrhea decreased after the mass meningococcal vaccination campaign carried out in 1989 to 1990,

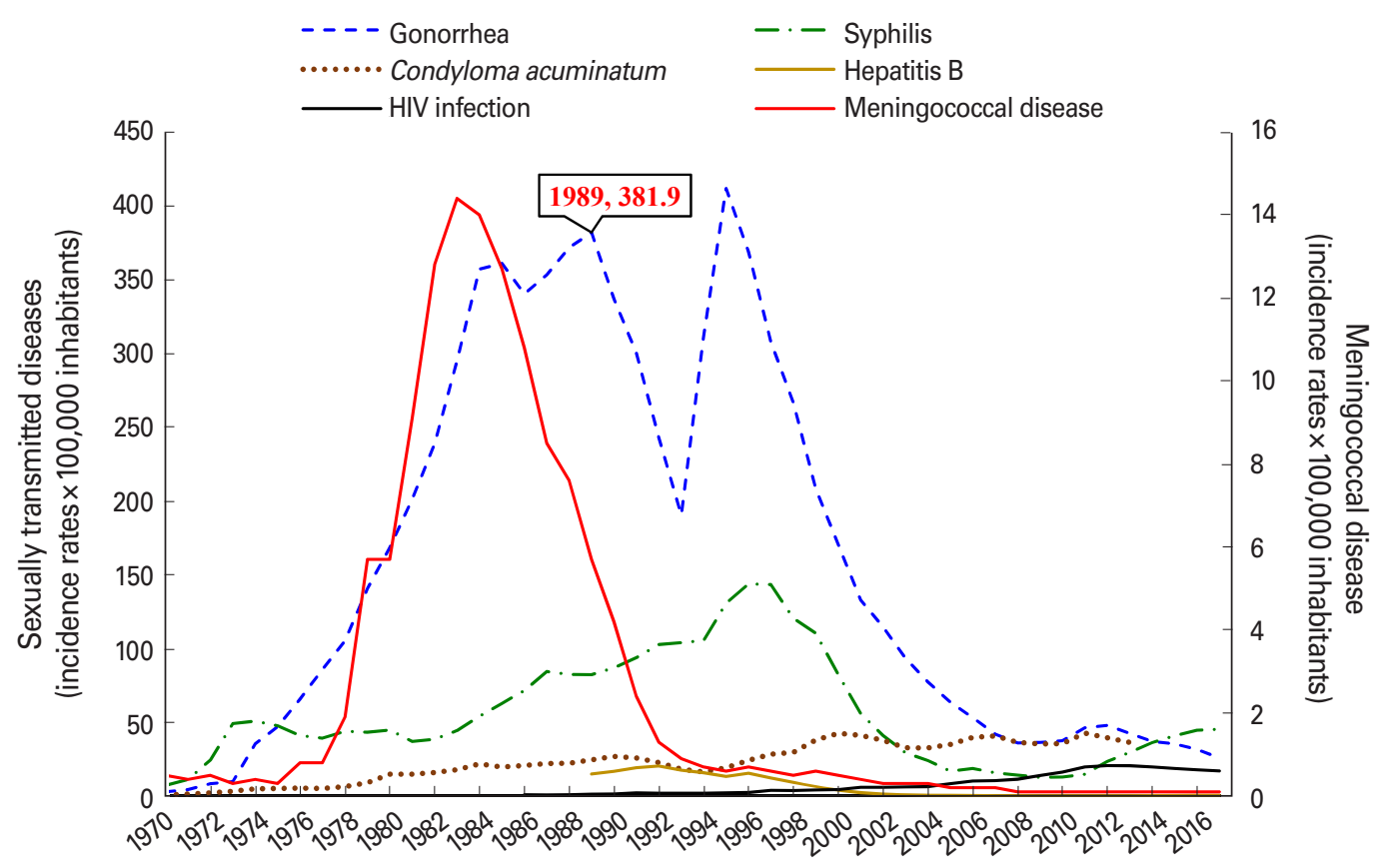

Fig. 1. Incidence rates of meningococcal disease and sexually transmitted diseases in Cuba. Data published in the Time Series [10] and the Cuban Health Statistical Yearbook [11] from 1970 to 2017 on the morbidity of meningococcal disease, gonorrhea and syphilis. Hepatitis B was reported between 1989 and 2017. HIV infection was registered in the Cuban statistical data and the Joint United Nations Programme on HIV and AIDS (UNAIDS) [12]. Condyloma acuminatum was analyzed with published data from 1970 to 2013. HIV, human immunodeficiency virus. 


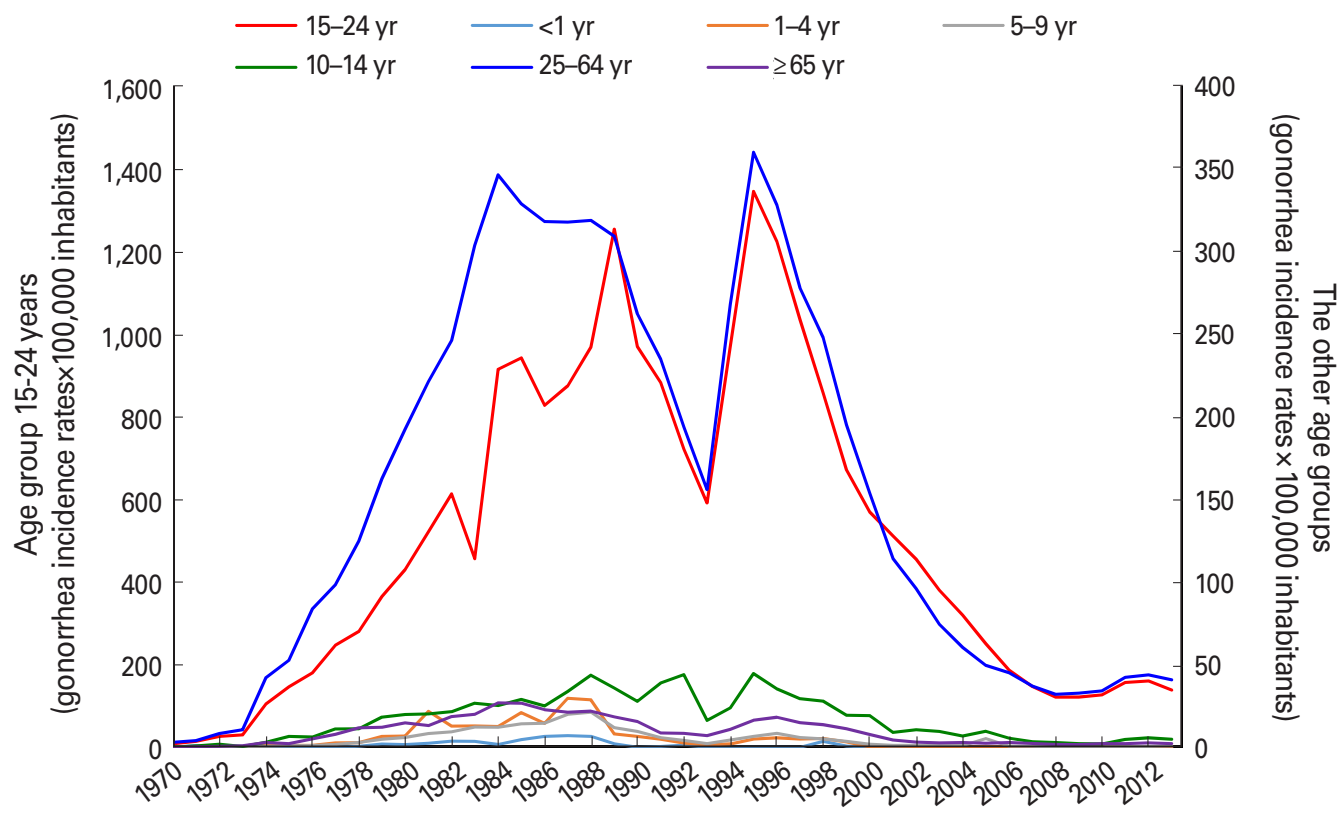

Fig. 2. Gonorrhea incidence rates by age groups. Data published in the Cuban Time Series from 1970 to 2013 [10].

Table 1. Incidence of sexually transmitted diseases and meningococcal disease in Cuba: 1989, 1993, and 2017

\begin{tabular}{lrrc}
\hline \multirow{2}{*}{ Disease } & \multicolumn{3}{c}{ Incidence rates $\times 100,000$ inhabitants } \\
\cline { 2 - 4 } & 1989 & 1993 & 2017 \\
\hline Meningococcal disease & 5.7 & 0.9 & 0.1 \\
Gonorrhea & 381.9 & 190.3 & 25.8 \\
Syphilis & 82.2 & 103.9 & 45.2 \\
Condyloma acuminatum & 24.5 & 18.1 & $36.3^{\text {a) }}$ \\
Hepatitis B & 14.9 & 17.5 & 0.5 \\
HIV infection & 1.2 & 1.8 & $17.0^{\mathrm{bl}}$ \\
\hline
\end{tabular}

HIV, human immunodeficiency virus.

al2013.

b) Joint United Nations Programme on HIV and AIDS (UNAIDS) [12]. 1989: beginning of meningococcal vaccination with VA-MENGOC-BC; 1993: 5 years after massvaccination campaign; 2017: last analyzed year.

although most sexually transmitted diseases continued to increase until 1993, 5 years after the start of vaccination. In 2017, the last year analyzed, the incidence of gonorrhea was markedly lower than that of 1989, when mass VA-MENGOC-BC vaccination began. Besides, it was lower than the incidence of syphilis that year, and the last report of condyloma acuminatum (Table 1) [12].

Gonorrhea was analyzed by age groups until 2013, the last year for which information was available. The highest morbidity rates were observed in the group of 15 to 24 years old, included in the mass-vaccination campaign, followed by the group of 25 to 64 years old, although with lower incidence rates. The remaining age groups presented much lower incidence rates (Fig. 2) [10].

\section{Discussion}

VA-MENGOC-BC vaccine has been successfully used in $\mathrm{Cu}$ ba and other countries to control epidemic meningococcal disease $[6,8,9]$. Meningococcal incidence rates dropped after the nationwide mass-vaccination campaign carried out in Cuba. Gonorrhea incidence was increasing until 1989, before the mass vaccination campaign. This increase was related to gradual improvement of gonorrhea diagnosis and surveillance of sexually transmitted diseases. Gonorrhea incidence rates also decreased after meningococcal vaccination $[7,10,11]$.

The decrease in gonorrhea incidence rates between 1989 and 1993 could be related to meningococcal B vaccination, especially taking into account the large morbidity increase in other sexually transmitted diseases during those years. Rates increased again until 1995, when they dropped again, which could be related to further meningococcal $B$ vaccination targeting unvaccinated high-risk groups. Vaccination coverage during the mass-vaccination campaign ranged from $75 \%$ to 95\% and progressively increased to $100 \%$ after 1995 [6-9]. On the other hand, initial vaccines from 3 months to 24 years of age have been gradually shifting to the sexually active groups, especially to the 25- to 64-year-old group, which is the second group with the highest gonorrhea morbidity rates $[3,7]$. Cor- 
relation coefficients were greater than 0.9 , indicating a very strong relationship between gonorrhea and vaccination.

The strong negative correlation detected between gonorrhea and syphilis from 1989 to 1993 indicates a relationship in which one variable increases (syphilis) as the other decreases (gonorrhea). On the other hand, syphilis has decreased considerably after 2000, which could be related to measures of health promotion and prevention of infection, and could also explain the reduction of gonorrhea morbidity rates. Nevertheless, gonorrhea incidence rates had already decreased before 2000; therefore, we cannot categorically rule out that the vaccine may have contributed to the protection against gonorrhea. Besides, it is striking that syphilis incidence has been higher than the incidence of gonorrhea since 2015 [10,11].

Hepatitis B dropped after 1992, when the recombinant hepatitis B surface antigen vaccine was introduced in the Cuban vaccination program, but a moderately negative relationship was found with respect to gonorrhea before vaccination.

The incidence of HIV infection is very low; however, it is increasing every year [10-13]. Similarly, a moderately negative relationship was found with respect to gonorrhea. A moderately negative relationship was also detected between gonorrhea and condyloma acuminatum. These results support the role of the meningococcal vaccine in gonorrhea prevention. Chlamydia infections was not taken into account, because diagnosis was not fully available, that is why there are no published data $[10,11]$.

The reduction of morbidity in the unvaccinated groups (Fig. 2) could be explained by herd immunity, taking into account that the 15- to 24-year-old group with the highest gonorrhea incidence was immunized during the massive vaccination campaign [10]. The unvaccinated population over 25 years old was $55.9 \%$ of the inhabitants during those years, and it was $47.4 \%$ in the 25 to 64 age group [10]. Herd immunity could be explained by the immune-protection acquired by sexual partners. On the other hand, individuals of the vaccinated age groups have been gradually shifting to the unvaccinated age groups. Thus, the immune-protected population has been growing with time.

VA-MENGOC-BC induced protection against gonorrhea could be moderate and short-lasting, considering the date of vaccination onset (1989) and that the lowest incidence rate was detected in 1993. The new increase of gonorrhea from 1993 to 1995 agrees with the epidemiological trends of gonorrhea and the other sexually transmitted diseases during the period from 1970 to 1995 . Nevertheless, further studies will be required to determine the duration and magnitude of the immune cross-protection against gonorrhea conferred by this vaccine.

Vaccine effectiveness for gonorrhea could not be estimated after the 1989 to 1990 mass vaccination campaign, because individuals with gonorrhea could not be separated into vaccinated or unvaccinated. That is why retrospective cohort or case-control studies could not be carried out.

Despite the strong positive correlation between vaccination and gonorrhea, we cannot categorically establish there is cross-protection. However, Neisseria meningitidis and Neisseria gonorrhoeae are two species that belong to the same genus. Therefore, they are closely related, and share between $80 \%$ and $90 \%$ of their genetic sequence $[1,2]$.

VA-MENGOC-BC is a vaccine based on very special OMV. More than one hundred proteins have been detected in them. The contribution of major proteins: PorA, PorB, Opa, OpcA, RmpM, FetA to their protein content is less than $65 \%$. Vesicles are especially lacking PorA (less than 20\%). However, there are enough to induce protection against the vaccine-type strain $[6,14]$.

High concentration of conserved proteins has been identified: NadA, fHbp, NMB0088, NMB1796, NMB0928, and a putative cell-binding factor protein. All of them induce widely cross-reactive antibodies against heterologous serogroup B strains [14], and very probably against Neisseria gonorrhoeae. Besides, other important minor components have been found: NspA, Tbp, HrpA, FbpA, PilQ, ATP synthases, bacterioferritins, heat shock proteins and ribosomal proteins. Some of them could also induce cross-reactive immune responses $[6,14]$. On the other hand, there are structural similarities between the PorB protein found in Neisseria meningitidis and Neisseria gonorrhoeae [15].

Some researchers have shown in human and animal models, that VA-MENGOC-BC induces the production of secretory IgA with high cross-reactivity against Neisseria gonorrhoeae $[6,7,16]$. However, additional studies must be carried out to detect specific cross-protective components against gonorrhea.

Cross-protection should not be surprising, the greatest success in vaccinology was the eradication of smallpox, using vaccinia virus as vaccine [17].

VA-MENGOC-BC was used with a two-dose scheme, and other studies have demonstrated that three doses of this vaccine, as well as other OMV vaccines, increase immunogenicity against heterologous strains of Neisseria meningitidis [6,14]; 
therefore, the use of three doses could also increase cross-protection against Neisseria gonorrhoeae. The MeNZB meningococcal vaccine used a three-dose scheme in New Zealand; its effectiveness against gonorrhea was $31 \%$ after the mass vaccination campaign during a meningococcal outbreak [4].

Meningococcal serogroup B OMV vaccines could induce moderate protection against gonorrhea. Although vaccination could be an additional measure, the promotion of safe sexual behavior and the systematic use of condoms are necessary to prevent gonorrhea and other sexually transmitted diseases [18].

One or more booster doses of VA-MENGOC-BC and other serogroup B OMV vaccines during adolescence, after priming in childhood, may contribute to protection against gonorrhea and increase herd immunity against Neisseria meningitidis. However, correlation alone does not imply causation, which is why research must be performed to detect shared proteins that may explain cross-reactivity and to evaluate the systemic and mucosal immunity provided by this vaccine. On the other hand, observational studies (case-control and cohort studies) should be carried out to estimate its clinical effectiveness against gonorrhea more accurately.

\section{ORCID}

Rolando Felipe Ochoa Azze https://orcid.org/0000-0002-80082944

\section{References}

1. Gonococcal Vaccine Development. Gonococcal vaccine development: lessons from group B meningococcal vaccines. EBioMedicine 2017;22:1.

2. Abbasi J. New hope for a gonorrhea vaccine. JAMA 2017; 318:894-5.

3. Petousis-Harris H. Impact of meningococcal group B OMV vaccines, beyond their brief. Hum Vaccin Immunother 2018;14:1058-63.

4. Petousis-Harris H, Paynter J, Morgan J, et al. Effectiveness of a group B outer membrane vesicle meningococcal vaccine against gonorrhoea in New Zealand: a retrospective case-control study. Lancet 2017;390:1603-10.

5. Seib KL. Gonorrhoea vaccines: a step in the right direction. Lancet 2017;390:1567-9.

6. Ochoa-Azze RF. Cross-protection induced by VA-MENGOCBC vaccine. Hum Vaccin Immunother 2018;14:1064-8.
7. Perez O, del Campo J, Cuello M, et al. Mucosal approaches in Neisseria Vaccinology. Vaccimonitor 2009;18:55-7.

8. Sierra GV, Campa HC, Varcacel NM, et al. Vaccine against group B Neisseria meningitidis: protection trial and mass vaccination results in Cuba. NIPH Ann 1991;14:195-207.

9. Sotolongo F, Campa C, Casanueva V, Fajardo EM, Cuevas IE, Gonzalez N. Cuban meningococcal BC vaccine: experiences \& contributions from 20 years of application. MEDICC Rev 2007;9:16-22.

10. Ministerio de Salud Publica (MINSAP). Direccion Nacional de Registros Medicos y Estadistica de Salud. Indicadores del estado de salud de la poblacion. Series de tiempo. Cuba 1970-2013 [Internet]. La Habana: MINSAP; c2014 [cited 2019 Feb 19]. Available from: http://files.sld.cu/dne/ files/2014/06/series-de-tiempo-cuba-1970_2013.pdf.

11. Ministerio de Salud Publica (MINSAP). Direccion Nacional de Registros Medicos y Estadistica de Salud. Anuario Estadistico de Salud 2017 [Internet]. La Habana: MINSAP; c2018 [cited 2019 Feb 19]. Available from: http://bvscuba. sld.cu/2017/11/20/anuario-estadistico-de-salud-de-cu$\mathrm{ba} /$.

12. Joint United Nations Programme on HIV and AIDS (UNAIDS). Country factsheets [Internet]. Geneva: UNAIDS; c2017 [cited 2019 Feb 19]. Available from: http://www.unaids.org/en/regionscountries/countries/cuba.

13. Gorry C. Cuba's national HIV/AIDS program. MEDICC Rev 2011;13:5-8.

14. Ochoa-Azze RF, Garcia-Imia L, Verez-Bencomo V. Effectiveness of a serogroup B and C meningococcal vaccine developed in Cuba. MEDICC Rev 2018;20:22-9.

15. Acevedo R, Bai X, Borrow R, et al. The Global Meningococcal Initiative meeting on prevention of meningococcal disease worldwide: epidemiology, surveillance, hypervirulent strains, antibiotic resistance and high-risk populations. Expert Rev Vaccines 2019;18:15-30.

16. Cuello M, Cabrera O, Acevedo R, et al. Nasal immunization with AFCol induces immune response to N. gonorrhoeae in mice. Vaccimonitor 2009;18:78-80.

17. Smith KA. Smallpox: can we still learn from the journey to eradication? Indian J Med Res 2013;137:895-9.

18. World Health Organization (WHO). WHO/RHR/16.09. Global health sector strategy on sexually transmitted infections. 2016-2021 [Internet]. Geneva: WHO; c2016 [cited 2019 Feb 19]. Available from: http://www.who.int/reproductivehealth/publications/rtis/ghss-stis/en/. 